\title{
Almost compatible X-microstructures in CuAlNi shape memory alloy
}

Received: 19 August 2011 / Accepted: 17 November 2011 / Published online: 4 December 2011 (C) The Author(s) 2011. This article is published with open access at Springerlink.com

\begin{abstract}
A systematic study of a specific martensitic microstructure, called the X-microstructure, is carried out with the focus on the CuAlNi shape memory alloy undergoing the cubic-to-orthorhombic transformation. The set of all crystallographically distinct candidate X-microstructures is determined, and it is shown that, according to the crystallographic theory of martensite, none of them is compatible. Almost compatible $\mathrm{X}$-microstructures, which involve elastic strains, are thus examined. These microstructures are searched in the neighborhood of all candidate $\mathrm{X}$-microstructures by minimizing the total elastic strain energy with respect to the microstructure parameters. Several low-energy X-microstructures are found, and it is shown that the total elastic strain energy correlates reasonably well with one of the indicators which characterize incompatibility of the corresponding candidate $\mathrm{X}$-microstructure.
\end{abstract}

Keywords Microstructure $\cdot$ Martensitic phase transformation $\cdot$ Shape memory alloys (SMA) · Energy minimization

\section{Introduction}

Shape memory alloys exhibit unique functional properties, such as shape recovery and pseudoelasticity, which originate from a reversible martensitic phase transformation. The transformation proceeds between a high temperature, high symmetry phase (austenite) and a low temperature, low symmetry phase (martensite). Due to the change of symmetry, several variants of martensite exist which have identical crystal structure but are mutually rotated. The need to accommodate the inelastic strains, which are associated with the change of crystal lattice during transformation, gives rise to the formation of martensitic microstructures, and the macroscopic behavior of shape memory alloys is governed by the evolution of these microstructures.

There are many special martensitic microstructures that are compatible, meaning that compatibility at the interfaces is achieved without elastic strains, possibly except in thin transition layers along the interfaces. The corresponding crystallographic theory of martensite has been first developed in a purely kinematic setting $[6,26]$, and subsequently, it has been cast in the framework of energy minimization [3], see also [12,17]. The crystallographic theory proved highly successful in predicting parameters of martensitic microstructures, see the monograph [5] for an overview.

As mentioned above, even in microstructures that are compatible, elastic strains may be present in the transition layers in order to accommodate the local incompatibility. Consider, for instance, the interface between

Communicated by Andreas Öchsner.

S. Stupkiewicz $(\bowtie) \cdot$ A. Górzyńska-Lengiewicz

Institute of Fundamental Technological Research (IPPT),

Polish Academy of Sciences, Pawińskiego 5B,

02-106 Warsaw, Poland

E-mail: sstupkie@ippt.gov.pl 


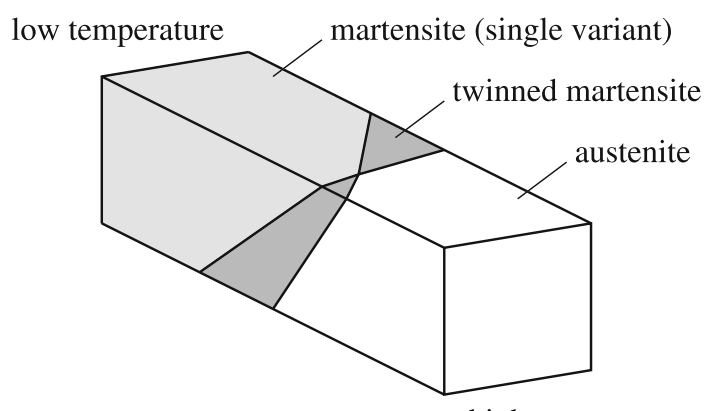

high temperature

Fig. $1 \mathrm{X}$-microstructure formed in a bar in temperature gradient

austenite and twinned martensite (fine laminate of two twin-related martensite variants) which is a typical martensitic microstructure. Such microstructure is compatible in the macroscopic (average) sense. However, a thin transition layer with non-zero elastic strains must develop along the interface because, locally, the individual variants and the austenite cannot form stress-free interfaces $[3,12-14,18]$. Local incompatibility in the transition layers is not further discussed in this work.

Let us note that the crystallographic theory rules out the microstructures that are not compatible, even if the respective compatibility conditions are only marginally violated, so that relatively small elastic strains would be sufficient to achieve compatibility. And, in fact, there exist martensitic microstructures that are observed experimentally and which are shown not to be compatible. It is thus of interest to study such almost compatible microstructures.

Balandraud et al. [1,2] have examined almost compatible wedge microstructures by virtually releasing the mid-rib interface. They have introduced an incompatibility indicator that measures the "mode-I" opening angle in this relaxed microstructure and have assumed that almost compatible wedges characterized by a small incompatibility indicator are most likely to form. For the cubic-to-tetragonal and cubic-to-orthorhombic transformations, they have determined the domains (low-energy neighborhoods) in the space of transformation stretch parameters, in which the incompatibility indicator is less than a prescribed small threshold value.

The X-microstructure is another example of an almost compatible microstructure that has been observed experimentally in InTl [4] and CuAlNi [22] (note that in this work we adopt the name "X-microstructure" rather than "X-interface" or " $\lambda$-interface" used in $[4,22])$. Experimentally, the $X$-microstructures are induced in single-crystal specimens transforming from a single variant of martensite back to austenite in a temperature gradient [22]. As a direct interface between the austenite and single variant of martensite would require very high elastic strains, the corresponding domains are separated by two domains of twinned martensite. The four interfaces involved meet at an intersection line and have an X-like appearance, see Fig. 1.

The X-microstructure in the cubic-to-tetragonal transformation (as in InTl) has been analyzed theoretically on the grounds of the crystallographic theory by Ruddock [19] who has shown that relevant compatibility conditions cannot be satisfied without elastic strains. Seiner et al. [21] analyzed the specific X-microstructure they have observed in CuAlNi [22]. Again, they have shown that, according to the crystallographic theory, the $\mathrm{X}$-microstructure is not compatible. They have also carried out a finite element analysis of the $\mathrm{X}$-microstructure formed within the specimen by assuming specific geometrical arrangement of the interfaces. The analysis has been further refined in [9] where optimal interface orientations have been determined that minimize the elastic strain energy. An important conclusion of the analysis in [9,21] is that the observed X-microstructures are not local energy minimizers as in all analyzed cases the energy can be reduced by moving the intersection line toward the specimen boundary (when the intersection line is on the boundary, the $\mathrm{X}$-microstructure degenerates to the V-microstructure introduced in Sect. 2.3 below).

In this paper, a systematic study of the X-microstructures is carried out with the focus on the CuAlNi alloy undergoing the cubic-to-orthorhombic transformation. The set of all crystallographically distinct candidate $\mathrm{X}$-microstructures is determined by considering the X-microstructure as an assembly of two V-microstructures. Two incompatibility indicators are introduced such that the candidate X-microstructure is compatible only when both indicators are equal to zero. Calculations carried out for CuAlNi indicate that none of the candidate $\mathrm{X}$-microstructures is compatible.

Almost compatible $\mathrm{X}$-microstructures are thus subsequently studied. The deformation gradient, uniform within each of the four domains forming the $\mathrm{X}$-microstructure, is multiplicatively decomposed into inelastic 
(transformation) and elastic parts. The total elastic strain energy is determined as a function of unknown microstructure and strain-like parameters and is next minimized with respect to all unknowns. It is shown that several X-microstructures are characterized by relatively low elastic strain energy, and the obtained microstructure parameters only slightly deviate from the parameters predicted by the crystallographic theory. It is also shown that the total elastic strain energy of these $\mathrm{X}$-microstructures correlates reasonably well with one of the indicators that characterize incompatibility of the corresponding candidate $\mathrm{X}$-microstructure.

Compared with the approach of Glatz et al. [9], the present model is a simplification in the sense that the effect of boundary conditions is neglected. However, being considerably simpler, the present approach is suitable for a systematic study of all 528 crystallographically distinct candidate X-microstructures. Furthermore, the volume fractions of martensite variants in the twinned domains are considered here as additional unknown parameters in the energy minimization scheme, while, in [9], the fixed values of the twin fractions are taken from the crystallographic theory.

\section{Compatible microstructures}

\subsection{Classical crystallographic theory of martensite}

Although the foundations of the crystallographic theory of martensite [3] are built on the energy minimization considerations, the practical applications of this theory rely on purely geometrical relationships. This is because compatible (i.e., stress-free) microstructures are only examined, so that the admissible deformation gradient $\mathbf{F}$ of an individual phase consists of the transformation stretch $\mathbf{U}$, known from crystallography, and a rotation $\mathbf{R}$, so that $\mathbf{F}=\mathbf{R U}$. Configuration of the undeformed austenite is usually adopted as a reference configuration, so the deformation gradient in the austenite is a unit tensor $\mathbf{F}=\mathbf{I}$, while the transformation stretches of different variants of martensite are mutually rotated, $\mathbf{U}_{J}=\mathbf{Q}_{I J} \mathbf{U}_{I} \mathbf{Q}_{I J}^{T}$, where $\mathbf{Q}_{I J}$ is a rotation belonging to the symmetry point group of austenite.

In a coherent phase transformation, the displacements are continuous along phase boundaries and this is expressed by the well-known kinematic compatibility condition,

$$
\mathbf{F}_{+}-\mathbf{F}_{-}=\mathbf{d} \otimes \boldsymbol{v}
$$

where $\mathbf{F}_{+}$and $\mathbf{F}_{-}$are the deformation gradients on both sides of the interface, $\boldsymbol{v}$ is the unit vector normal to the interface, and $\mathbf{d}$ is a vector.

Considering a specific microstructure, the deformation gradients on both sides of the interface are typically known (up to a rotation), and the goal is to find the orientation of the interface, if it exists. For that purpose, the procedure based on Proposition 4 in [3] can be used, as summarized below. A symmetric matrix $\mathbf{C}$ is first calculated according to

$$
\mathbf{C}=\mathbf{F}_{-}^{-T} \mathbf{F}_{+}^{T} \mathbf{F}_{+} \mathbf{F}_{-}^{-1},
$$

and solution to Eq. (1) exists if and only if $\Lambda_{2}=1$, where $\Lambda_{i}$ are ordered eigenvalues of $\mathbf{C}, \Lambda_{1} \leq \Lambda_{2} \leq \Lambda_{3}$. If $\Lambda_{2}=1$, then there are exactly two solutions, and the corresponding vectors $\boldsymbol{v}$ and $\mathbf{d}$, as well as the unknown rotation in $\mathbf{F}_{+}$, are determined in terms of the eigenvalues and eigenvectors of $\mathbf{C}$. For details, refer to [3,5].

Below the equations of the crystallographic theory are presented for the twinning and habit planes, which are the classical applications of the theory, and the details can be found, e.g., in [5,11]. Less classical microstructures relevant to the analysis of the X-microstructure are treated in the subsequent subsections. While the approach is general, the detailed analysis is carried out for the cubic-to-orthorhombic phase transformation, and in particular for the CuAlNi shape memory alloy ( $\beta_{1} \rightarrow \gamma_{1}^{\prime}$ transformation).

\subsubsection{Twin $(M-M)$ interface}

Consider an interface separating two stress-free variants of martensite ( $\mathrm{M}-\mathrm{M}$ interface, or twinning plane). Denoting by $\mathbf{U}_{I}$ and $\mathbf{U}_{J}$ the transformation stretches of the two variants, the kinematic compatibility condition (1) is written in the form of the twinning equation,

$$
\mathbf{R U}_{I}-\mathbf{U}_{J}=\mathbf{a} \otimes \mathbf{l},
$$

where the unknowns are the twinning plane normal $\mathbf{l}$, twinning shear vector $\mathbf{a}$, and twin rotation $\mathbf{R}$. 
Cubic-to-orthorhombic transformation In the cubic-to-orthorhombic transformation, there are six variants of martensite. The components of the transformation stretch tensor of the first variant are

$$
U_{1}=\left(\begin{array}{ccc}
\frac{\alpha+\gamma}{2} & 0 & \frac{\alpha-\gamma}{2} \\
0 & \beta & 0 \\
\frac{\alpha-\gamma}{2} & 0 & \frac{\alpha+\gamma}{2}
\end{array}\right),
$$

where $\alpha, \beta$ and $\gamma$ are stretch parameters that depend on the lattice parameters of austenite and martensite phases. In case of the $\beta_{1} \rightarrow \gamma_{1}^{\prime}$ transformation in CuAlNi, the stretch parameters are $\alpha=1.0619, \beta=0.9178$ and $\gamma=1.0230$ [5]. Note that the numbering of variants used in this work is consistent with that used, for instance, in [1,5], but different than that used in [11,20,22]. Here and below, the components of vectors and tensors are provided in the cubic basis of austenite.

In case of the cubic-to-orthorhombic transformation, solutions to the twinning equation (3) exist for any pair of variants $(I, J)$. In case of the $(1,2)$ pair and the crystallographically equivalent pairs, namely the $(3,4)$ and $(5,6)$ pairs, the two solutions are the compound twins with rational twinning plane normals. In case of the $(1,3)$ pair and all the remaining pairs, which are crystallographically equivalent to $(1,3)$, the first solution is a Type I twin and the second solution is a Type II twin with, respectively, rational and irrational twinning plane normals.

\subsubsection{Austenite-twinned martensite (A-MM) interface}

Existence of the interface between austenite and a single variant of martensite, with both phases in stress-free conditions, would require the following compatibility condition to be satisfied,

$$
\hat{\mathbf{R}} \mathbf{U}_{I}-\mathbf{I}=\mathbf{b} \otimes \mathbf{m}
$$

with unknown $\mathbf{m}, \mathbf{b}$ and $\hat{\mathbf{R}}$. The austenite-single martensite variant interface is possible if the lattice parameters satisfy special conditions. ${ }^{1}$ This is not the case of the majority of known shape memory alloys, in which compatibility at the austenite-martensite interface is obtained by twinning in martensite (random stacking faulting is another known mechanism [10,23]).

The compatibility condition formulated for the interface separating the austenite and twinned martensite takes the form of the following habit plane equation,

$$
\hat{\mathbf{R}}\left(\lambda \mathbf{R} \mathbf{U}_{I}+(1-\lambda) \mathbf{U}_{J}\right)-\mathbf{I}=\mathbf{b} \otimes \mathbf{m},
$$

where the unknowns are the twin fraction $\lambda$, habit plane normal $\mathbf{m}$, shape strain vector $\mathbf{b}$ and rotation $\hat{\mathbf{R}}$, while the rotation $\mathbf{R}$ satisfies the twinning equation (3). We note that the first term in equation (6) is the average deformation gradient of the laminate consisting of two twin-related variants of martensite. Thus, the habit plane equation (6) enforces compatibility and stress-free conditions at the macro-scale, while elastic strains are necessarily present within a transition layer along the interface in order to enforce compatibility of individual phases at the micro-scale. The energy of elastic micro-strains and the morphology of the transition layer can be predicted using the approach proposed in [14,24].

Solution of the habit plane equation (6) proceeds by finding the twin fraction $0<\lambda<1$ for which the condition $\Lambda_{2}=1$ is satisfied for the relevant matrix $\mathbf{C}$ constructed according to Eq. (2). If such $\lambda$ exists, then the remaining unknowns can be found by applying Proposition 4 in [3], which yields two solutions for a fixed twinning mode and fixed twin fraction $\lambda$. If the solution to Eq. (6) exists for $\lambda=\lambda^{*}$ then it also exists for $\lambda=1-\lambda^{*}$.

Cubic-to-orthorhombic transformation In the cubic-to-orthorhombic transformation in CuAlNi, compatible A-MM interfaces are only possible for the martensite variant pairs of the $(1,3)$ type. For each variant pair, there are $2 \times 2 \times 2=8$ austenite-twinned martensite microstructures (two twinning modes $\times$ two twin fractions $\times$ two A-MM interfaces), and the total of 96 distinct microstructures considering the 12 crystallographically equivalent variant pairs.

\footnotetext{
1 The search for the materials that would form direct austenite-single martensite variant interfaces is currently an active area of research, cf. $[8,28]$, aimed at finding low-hysteresis shape memory alloys.
} 


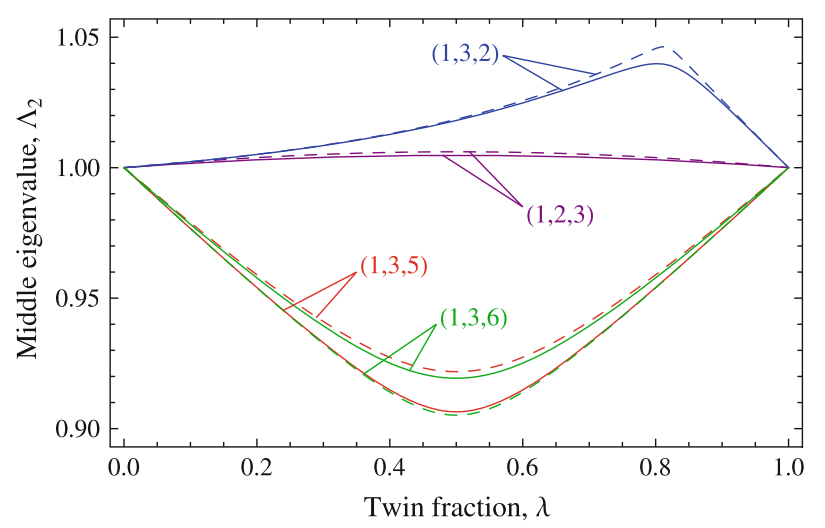

Fig. 2 Middle eigenvalue $\Lambda_{2}$ for M-MM interfaces in the case of $K \neq I$ and $K \neq J$ (solid lines-type I twinning of variants $I$ and $J$, dashed lines-type II twinning)

\subsection{Martensite-twinned martensite (M-MM) interface}

Consider now an interface between a finely twinned martensite (variants $I$ and $J$ ) and a single variant of martensite (variant $K$ ). The corresponding compatibility condition reads

$$
\tilde{\mathbf{R}}\left(\lambda \mathbf{R} \mathbf{U}_{I}+(1-\lambda) \mathbf{U}_{J}\right)-\mathbf{U}_{K}=\tilde{\mathbf{c}} \otimes \mathbf{n},
$$

where the rotation $\mathbf{R}$ satisfies the twinning equation (3), while the twin fraction $\lambda$ is arbitrary $(0<\lambda<1)$. As in the case of the A-MM interface, Eq. (7) is a macroscopic compatibility condition formulated for the average deformation gradient in the twinned martensite. Microstructures of this kind have been studied in detail in [7] for the case of compound twins $(I, J, K=1,2)$ in CuAlNi.

If only two martensite variants are involved (i.e., if we have $K=I$ or $K=J$ ), then a trivial solution to Eq. (7) is found immediately with $\mathbf{n}=\mathbf{l}$. We also have $\tilde{\mathbf{c}}=\lambda \mathbf{a}$ and $\tilde{\mathbf{R}}=\mathbf{I}$ if $K=J$, and $\tilde{\mathbf{c}}=-(1-\lambda) \mathbf{R}^{T} \mathbf{a}$ and $\tilde{\mathbf{R}}=\mathbf{R}^{T}$ if $K=I$. Furthermore, Proposition 4 in [3] guarantees that there is also the second solution. The non-trivial solution can be found by applying Proposition 4 in [3] or using the result in Appendix $\mathrm{H}$ in [7]. Clearly, the non-trivial solution $(\mathbf{n}, \tilde{\mathbf{c}}, \tilde{\mathbf{R}}$ ) depends on the twin fraction $\lambda$ (which is arbitrary here, $0<\lambda<1$ ). The above result is general, as we have only assumed that variants $I$ and $J$ form a twin. The M-MM interface is then found to be approximately perpendicular to the twinning plane.

In the case of $K \neq I$ and $K \neq J$, the algebra becomes too difficult to arrive at a general result. Hence, only numerical results are presented below for the cubic-to-orthorhombic transformation in CuAlNi.

Cubic-to-orthorhombic transformation Consider first the non-trivial M-MM interface in CuAlNi for $K=J$ (the case of $K=I$ is fully equivalent). In the limit of $\lambda \rightarrow 0$, the angle between the twinning plane normal $\mathbf{I}$ and the non-trivial $\mathrm{M}-\mathrm{MM}$ interface normal $\mathbf{n}$ is $87.9^{\circ}$ for compound twins, $82.7^{\circ}$ for Type I twins and $82.6^{\circ}$ for Type II twins. At the same time, for $\lambda \rightarrow 1, \mathbf{l}$ and $\mathbf{n}$ are exactly perpendicular (the case of $\lambda=1$ corresponds to the exact twinning relation of pure variants $I$ and $J$ ).

In the case of $K \neq I$ and $K \neq J$, it has been found that Eq. (7) has no solution for $0<\lambda<1$. This has been verified numerically by examining the middle eigenvalue $\Lambda_{2}$ of the matrix $\mathbf{C}$ derived according to Eq. (2) for the compatibility condition in Eq. (7). Figure 2 presents the middle eigenvalue $\Lambda_{2}$ as a function of the twin fraction $\lambda$ for all crystallographically distinct triples $(I, J, K)$. It is seen that the condition $\Lambda_{2}=1$ is not satisfied except in the limiting cases of $\lambda=0$ and $\lambda=1$, which correspond to simple twinning of two variants.

\subsection{V-microstructure}

Let us define a V-microstructure formed within a specimen of finite dimensions or within a half-space, as shown in Fig. 3. The domain $\Omega_{2}$ of twinned martensite $\left(M_{I}+M_{J}\right)$ separates the austenite $(A)$ in domain $\Omega_{1}$ and a pure variant of martensite $\left(M_{I}\right)$ in domain $\Omega_{3}$. The normal to the A-MM interface (habit plane), denoted 


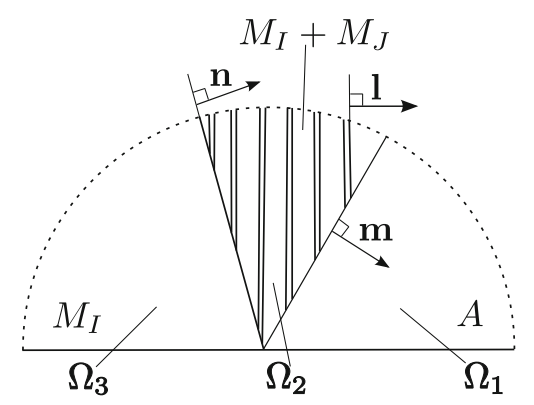

Fig. 3 Sketch of the V-microstructure (note that $\mathbf{l}$ is not necessarily coplanar with $\mathbf{m}$ and $\mathbf{n}$ )

by $\mathbf{m}$, points to the domain $\Omega_{1}$ and the normal the M-MM interface, denoted by $\mathbf{n}$, points to the domain $\Omega_{2}$. The orientation of the intersection line is specified by a unit vector $\mathbf{t}$,

$$
\mathbf{t}=\frac{\mathbf{m} \times \mathbf{n}}{\|\mathbf{m} \times \mathbf{n}\|} .
$$

In a specimen of finite dimensions, the intersection line reduces to a point on the specimen boundary, unless the free surface has a special orientation. A special orientation of the free surface is also necessary if the body in the reference configuration (austenite) occupies a half-space.

We notice that a stress-free V-microstructure is always possible whenever an A-MM interface exists. This is because M-MM interfaces involving two variants of martensite are always possible, as discussed above (the case of $K=I$ or $K=J$ ). At the same time, a stress-free $\mathrm{V}$-microstructure involving three distinct variants of martensite is not possible because the corresponding M-MM interface is not possible (at least in the case of the cubic-to-orthorhombic transformation in $\mathrm{CuAlNi}$, as discussed above).

The $\mathrm{V}$-microstructure is introduced here as an auxiliary microstructure that will be used later in the analysis of the X-microstructure. However, the V-microstructures themselves are admissible and may form in specimens of finite dimensions, e.g., in a prism-shaped single-crystal specimen subjected to temperature gradient [15].

The deformation gradients in the $\mathrm{V}$-microstructure are

$$
\mathbf{F}_{1}=\mathbf{I}, \quad \mathbf{F}_{2}=\mathbf{I}+\mathbf{b} \otimes \mathbf{m}, \quad \mathbf{F}_{3}=\mathbf{I}+\mathbf{b} \otimes \mathbf{m}+\mathbf{c} \otimes \mathbf{n},
$$

where $\mathbf{m}$ and $\mathbf{b}$ satisfy the habit plane equation (6), with the rotation $\mathbf{R}$ satisfying the twinning equation (3). The M-MM interface equation (7) is rewritten here, so that the rotation $\mathbf{R}_{3}$, such that $\mathbf{F}_{3}=\mathbf{R}_{3} \mathbf{U}_{I}$, is explicitly involved, namely

$$
\hat{\mathbf{R}}\left(\lambda \mathbf{R} \mathbf{U}_{I}+(1-\lambda) \mathbf{U}_{J}\right)-\mathbf{R}_{3} \mathbf{U}_{I}=\mathbf{c} \otimes \mathbf{n},
$$

where $\hat{\mathbf{R}}$ and $\lambda$ satisfy the habit plane equation (6) and, comparing to Eq. (7), we have $\mathbf{U}_{K}=\mathbf{U}_{I}, \tilde{\mathbf{R}}=\mathbf{R}_{3}^{T} \hat{\mathbf{R}}$ and $\tilde{\mathbf{c}}=\mathbf{R}_{3}^{T} \mathbf{c}$. Note that if $\mathbf{n}=\mathbf{l}$ (trivial M-MM interface) then $\mathbf{R}_{3}=\hat{\mathbf{R}} \mathbf{R}$, i.e., the rotations of variant $I$ in domains $\Omega_{3}$ and $\Omega_{2}$ are identical.

Cubic-to-orthorhombic transformation It is of interest for our future considerations to examine the number of crystallographically distinct $\mathrm{V}$-microstructures in CuAlNi. Consider thus a representative pair of martensite variants $(I, J)$ of the $(1,3)$ type, for which the austenite-twinned martensite interface exists. There are two twinning systems (Type I and Type II twins), and for each of them there are two twin fractions $\lambda=\lambda_{I}=1-\lambda_{J}$ $(\lambda \approx 0.3$ and $\lambda \approx 0.7)$. Now, for each twinning system and for each twin fraction, there are two A-MM interfaces and two M-MM interfaces, so that altogether there are $2 \times 2 \times 2 \times 2=16 \mathrm{~V}$-microstructures. Crystallographically equivalent V-microstructures are obtained by applying rotations from the symmetry point group of austenite.

Note that the geometric arrangement of the phases is not considered here. Two cases can be considered with $\mathbf{m} \cdot \mathbf{n}>0$ or $\mathbf{m} \cdot \mathbf{n}<0$, corresponding to, respectively, an acute or obtuse angle between the A-MM interface and the M-MM interface. This will be discussed in more detail later. 


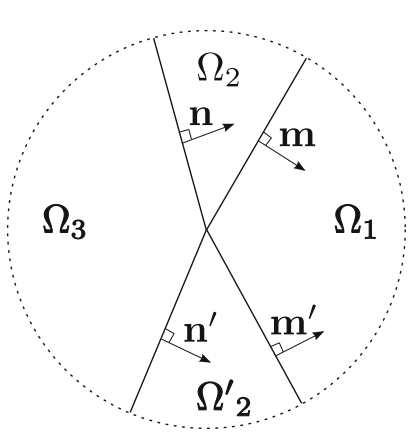

(a)

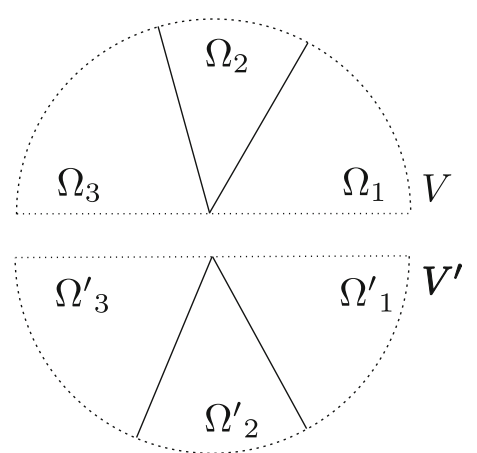

(b)

Fig. 4 Sketch of the X-microstructure

\subsection{X-microstructure}

The X-microstructure consists of an austenite domain $\Omega_{1}$, a single martensite variant domain $\Omega_{3}$ and two twinned martensite domains $\Omega_{2}$ and $\Omega_{2}^{\prime}$, separated by two A-MM interfaces and two M-MM interfaces, cf. Fig. 4a. The X-microstructure involves thus an ordered triple $(I, J, K)$ of martensite variants. The pairs $(I, J)$ and $(I, K)$ must form stress-free A-MM interfaces (e.g., be of the $(1,3)$ type in the case of the cubic-to-orthorhombic transformation in CuAlNi).

It is convenient to consider the $\mathrm{X}$-microstructure as an assembly of two admissible V-microstructures, cf. Fig. 4b. Let us denote by $V$ and $V^{\prime}$ the V-microstructures formed by the $(I, J)$ and $(I, K)$ pairs, respectively. There are two necessary conditions for the existence of a stress-free X-microstructure [21], see also [19] for an alternative formulation. Firstly, the intersection lines must have the same orientation and, secondly, the deformation gradients $\mathbf{F}_{3}=\mathbf{R}_{3} \mathbf{U}_{I}$ and $\mathbf{F}_{3}^{\prime}=\mathbf{R}_{3}^{\prime} \mathbf{U}_{I}$ must be equal, thus

$$
\mathbf{t} \times \mathbf{t}^{\prime}=0, \quad \mathbf{R}_{3}=\mathbf{R}_{3}^{\prime},
$$

where the quantities without and with the prime correspond to $V$ and $V^{\prime}$, respectively.

There is also an additional condition of geometrical feasibility, namely that the vector quadruple ( $\pm \mathbf{m}, \pm \mathbf{n}, \pm \mathbf{n}^{\prime}, \pm \mathbf{m}^{\prime}$ ) can be arranged in a clockwise or anticlockwise manner (within the plane normal to $\mathbf{t}=\mathbf{t}^{\prime}$ ). However, it follows from elementary considerations that the four domains can be arranged in a geometrically feasible manner except if $\mathbf{m}= \pm \mathbf{m}^{\prime}$ and $\mathbf{n}= \pm \mathbf{n}^{\prime}$. Accordingly, two identical V-microstructures cannot be assembled into a X-microstructure. For two distinct V-microstructures, we have $\mathbf{m} \neq \pm \mathbf{m}^{\prime}$ and the above geometrical condition does not intervene.

The necessary conditions (11) will be checked numerically for candidate (potential) X-microstructures formed by all pairs of V-microstructures. In order to quantitatively characterize compatibility of a candidate $\mathrm{X}$-microstructure, we introduce two incompatibility indicators, cf. [21], each corresponding to one of the necessary conditions (11),

$$
\phi=\arcsin \left|\mathbf{t} \times \mathbf{t}^{\prime}\right|, \quad \psi=\arccos \left(\frac{\operatorname{tr}\left(\mathbf{R}_{3}^{\prime} \mathbf{R}_{3}^{T}\right)-1}{2}\right) .
$$

Here, $\phi$ is the angle between the intersection lines of the two V-microstructures, and $\psi$ is the angular distance between rotations $\mathbf{R}_{3}$ and $\mathbf{R}_{3}^{\prime}$. Clearly, the necessary conditions (11) are satisfied if $\phi=\psi=0$.

Note that the incompatibility indicator $\psi$ defined above is not equivalent to the incompatibility indicator $\psi$ introduced by Balandraud et al. [1] in the context of wedge microstructures, though formally it bears some similarity.

Cubic-to-orthorhombic transformation We start by determining the set of crystallographically distinct pairs of V-microstructures. We remind that the variant pairs $(I, J)$ and $(I, K)$ are of the $(1,3)$ type. There are thus four crystallographically distinct ordered variant triples $(I, J, K)$, namely $(1,3,3),(1,3,4),(1,3,5)$ and $(1,3,6)$, and all the remaining feasible triples (and the corresponding candidate X-microstructures) can be obtained by applying the symmetry point group of the (cubic) austenite. 


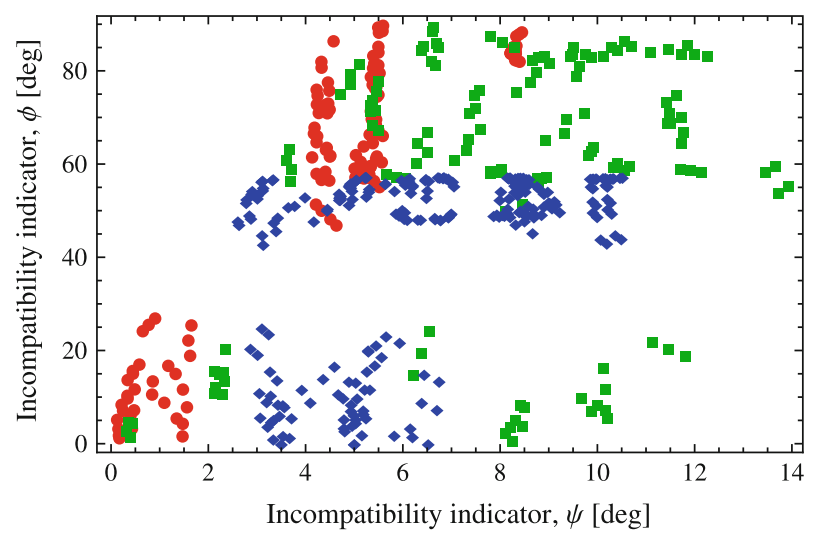

Fig. 5 Incompatibility indicators $\phi$ and $\psi$ computed for all crystallographically distinct pairs of V-microstructures, i.e., for all candidate $\mathrm{X}$-microstructures

Considering that a candidate $\mathrm{X}$-microstructure is formed by two V-microstructures, with $16 \mathrm{~V}$-microstructures possible for each variant pair, there are $16 \times 16=256$ combinations of $\mathrm{V}$-microstructures to be considered for each variant triple. However, the $\left(V_{i}, V_{j}^{\prime}\right)$ pair, $i, j=1, \ldots 16$, is equivalent to the $\left(V_{j}, V_{i}^{\prime}\right)$ pair, so that there are 136 crystallographically distinct combinations such that $i \geq j$. Furthermore, in the case of the $(1,3,3)$ triple, the pairs $\left(V_{i}, V_{i}^{\prime}\right)$ of two identical V-microstrucures are ruled out by the condition of geometrical feasibility discussed above, though they automatically satisfy the necessary conditions (11). Hence, only the combinations such that $i>j$ are considered for the $(1,3,3)$ triple, and there are 120 such combinations. Concluding, there are 120 crystallographically distinct pairs of V-microstructures for the $(1,3,3)$ triple and 136 pairs for the $(1,3,4),(1,3,5)$, and $(1,3,6)$ triples, so that the total number of candidate $\mathrm{X}$-microstructures is $120+3 \times 136=528$.

The calculations carried out for the CuAlNi alloy indicate that the necessary conditions (11) are not satisfied by any of the 528 crystallographically distinct combinations of $\mathrm{V}$-microstructures. This is illustrated in Fig. 5 which presents the values of the incompatibility indicators $\phi$ and $\psi$ computed for all crystallographically distinct pairs of V-microstructures. Here, each point on the $(\phi, \psi)$-plane corresponds to one candidate $\mathrm{X}$-microstructure. The red dots in Fig. 5 indicate candidate microstructures with twin fractions of variants $J$ and $K$ satisfying $\lambda_{J}=1-\lambda \approx 0.3$ and $\lambda_{K}^{\prime}=1-\lambda^{\prime} \approx 0.3$, respectively. Thus, variant $I$, which occupies domain $\Omega_{3}$, also prevails in the twinned domains $\Omega_{2}$ and $\Omega_{2}^{\prime}$. This situation seems physically more realistic as compared with the opposite case ( $\lambda_{J} \approx 0.7$ and $\lambda_{K}^{\prime} \approx 0.7$, green squares) and the mixed case (either $\lambda_{J} \approx 0.7$ or $\lambda_{K}^{\prime} \approx 0.7$, blue diamonds), particularly in reference to shape recovery experiments of [22].

It is seen that the condition $\phi=\psi=0$ is not satisfied by any of the candidate X-microstructures, and hence, a stress-free X-microstructure is not possible in this alloy. However, in some cases, the incompatibility indicators are rather close to zero. This suggests that, in these cases, the compatibility might be achieved at relatively low elastic strains. This possibility is investigated in the next section.

Remark 1 Seiner et al. [22] have observed two types of X-microstructures, which they called "X-interface" and " $\lambda$-interface". In the "X-interface", both M-MM interfaces are approximately parallel to the corresponding twinning planes, while in the " $\lambda$-interface" one of the M-MM interfaces is not parallel to the corresponding twinning plane. As discussed in Sect. 2.2, the M-MM interface can either be parallel or approximately perpendicular to the twinning planes. Both types of $\mathrm{M}-\mathrm{MM}$ interfaces are thus covered by the analysis above.

\section{Almost compatible $\mathrm{X}$-microstructures}

As shown above, compatible (stress-free) X-microstructures cannot form in the CuAlNi alloy. Thus, the experimentally observed X-microstructures [22] must involve elastic strains, so that the compatibility conditions are satisfied. Our aim, here, is to determine microstructure parameters, such as interface normals and twin fractions, by minimizing the elastic strain energy in the $\mathrm{X}$-microstructure.

We assume here that the deformation gradients, comprising both the transformation and elastic parts, are constant within each domain $\Omega_{i}$, cf. Fig. 4. By assuming that the deformation gradient is piecewise-constant, we neglect the effect of boundary conditions in a specimen (e.g., a bar), so that the analysis applies to the 
neighborhood of the intersection line. A finite element study including the influence of free boundary, but focused on a specific X-microstructure observed in a CuAlNi bar [22], is reported in [21].

The effects related to temperature gradient and non-isothermal conditions are not included in the present model. In the experiments reported in [22], the X-microstructures were induced by temperature gradient (formation of an austenite nucleus in a bar transformed to a single variant of martensite), and its subsequent propagation was driven either by temperature gradient or by controlling the temperature in a warm bath. While the temperature gradient is the main driving force for nucleation and propagation of the $\mathrm{X}$-microstructures, its effect on the microstructure parameters and elastic strain energy is expected to be negligible, as the thermal strains are small compared with the transformation strains.

\subsection{Elastic strains and compatibility conditions}

Multiplicative split of the deformation gradient into an elastic part $\mathbf{F}^{e}$ and an inelastic (transformation) part $\mathbf{F}^{t}$ is assumed,

$$
\mathbf{F}=\mathbf{F}^{e} \mathbf{F}^{t}
$$

The configuration of stress-free austenite is adopted as the reference configuration, thus $\mathbf{F}^{t}=\mathbf{I}$ in the austenite and $\mathbf{F}^{t}=\mathbf{U}_{I}$ in the $I$-th variant of martensite, where $\mathbf{U}_{I}$ is the corresponding transformation stretch tensor. Hence, the transformation part $\mathbf{F}^{t}$ is known from crystallography, while the elastic part $\mathbf{F}^{e}$ comprises unknown elastic stretch and rigid-body rotation.

The constitute law is specified by the elastic strain energy function $W(\mathbf{F})$, per unit volume in the reference configuration. An anisotropic St. Venant-Kirchhoff model is adopted in the form

$$
W(\mathbf{F})=\frac{1}{2}\left(\operatorname{det} \mathbf{F}^{t}\right) \mathbf{E}^{e} \cdot \mathbf{L} \mathbf{E}^{e}, \quad \mathbf{E}^{e}=\frac{1}{2}\left(\mathbf{F}^{e T} \mathbf{F}^{e}-\mathbf{I}\right),
$$

where $\mathbf{L}$ is the fourth-order elastic moduli tensor and $\mathbf{E}^{e}$ is the elastic Green strain tensor; both $\mathbf{L}$ and $\mathbf{E}^{e}$ refer to the intermediate stress-free configuration. Due to anisotropy, the elastic moduli tensors are different in each phase (and martensite variant). They possess usual symmetries and are positive definite. In the computations reported in Sect. 4, the elastic constants of cubic austenite and orthorhombic martensite of CuAlNi alloy are taken from [25,27], see also [20].

At each interface, the kinematic compatibility condition (1) is now enforced for the total deformation gradient, including the elastic part. Additionally, the stresses must satisfy the following compatibility condition, stemming from the requirement of mechanical equilibrium,

$$
\left(\mathbf{S}_{+}-\mathbf{S}_{-}\right) \boldsymbol{v}=\mathbf{0},
$$

where $\mathbf{S}=\partial W / \partial \mathbf{F}$ is the first Piola-Kirchhoff stress, and we have $\mathbf{S}=(\operatorname{det} \mathbf{F}) \boldsymbol{\sigma} \mathbf{F}^{-T}$, where $\boldsymbol{\sigma}$ is the Cauchy stress.

The compatibility conditions (1) and (15) must hold at all A-MM, M-MM, and M-M interfaces in the $\mathrm{X}$-microstructure. Below, we explicitly specify the kinematic compatibility conditions. Discussion of the stress-equilibrium conditions is deferred till the end of Sect. 3.2.

Kinematic compatibility conditions formulated for the A-MM and M-MM interfaces read

$$
\mathbf{F}_{2}-\mathbf{F}_{1}=\mathbf{b} \otimes \mathbf{m}, \quad \mathbf{F}_{3}-\mathbf{F}_{2}=\mathbf{c} \otimes \mathbf{n}, \quad \mathbf{F}_{2}^{\prime}-\mathbf{F}_{1}=\mathbf{b}^{\prime} \otimes \mathbf{m}^{\prime}, \quad \mathbf{F}_{3}-\mathbf{F}_{2}^{\prime}=\mathbf{c}^{\prime} \otimes \mathbf{n}^{\prime} .
$$

We note that the above conditions enforce the following constraint on the corresponding normal and shape strain vectors,

$$
\mathbf{b} \otimes \mathbf{m}+\mathbf{c} \otimes \mathbf{n}-\left(\mathbf{b}^{\prime} \otimes \mathbf{m}^{\prime}+\mathbf{c}^{\prime} \otimes \mathbf{n}^{\prime}\right)=\mathbf{0} .
$$

The deformation gradients $\mathbf{F}_{2}$ and $\mathbf{F}_{2}^{\prime}$ in the twinned martensite domains $\Omega_{2}$ and $\Omega_{2}^{\prime}$, respectively, are the average deformation gradients, namely

$$
\mathbf{F}_{2}=\lambda \mathbf{F}_{21}+(1-\lambda) \mathbf{F}_{22}, \quad \mathbf{F}_{2}^{\prime}=\lambda^{\prime} \mathbf{F}_{21}^{\prime}+\left(1-\lambda^{\prime}\right) \mathbf{F}_{22}^{\prime},
$$

where $\lambda$ and $\lambda^{\prime}$ are the corresponding volume fractions of the martensite variant $I$. The local deformation gradients $\mathbf{F}_{21}, \mathbf{F}_{22}, \mathbf{F}_{21}^{\prime}$ and $\mathbf{F}_{22}^{\prime}$ must satisfy the kinematic compatibility conditions at M-M interfaces, namely

$$
\mathbf{F}_{22}-\mathbf{F}_{21}=\mathbf{a} \otimes \mathbf{l}, \quad \mathbf{F}_{22}^{\prime}-\mathbf{F}_{21}^{\prime}=\mathbf{a}^{\prime} \otimes \mathbf{l}^{\prime} .
$$


3.2 Minimization of elastic strain energy

The $\mathrm{X}$-microstructure is defined by the set $\mathcal{M}$ of microstructure parameters,

$$
\mathcal{M}=\left\{\mathbf{m}, \mathbf{m}^{\prime}, \mathbf{n}, \mathbf{n}^{\prime}, \mathbf{l}, \mathbf{l}^{\prime}, \lambda, \lambda^{\prime}\right\}
$$

where all the normal vectors have unit length, and the $\mathrm{A}-\mathrm{MM}$ and $\mathrm{M}-\mathrm{MM}$ interface normals are coplanar.

It is convenient to represent each unit vector in $\mathcal{M}$ by a pair of rotation angles $\left(\theta_{i}, \varphi_{i}\right)$, so that the corresponding unit length constraints are satisfied automatically. Further, we relax the coplanarity condition and introduce the set $\mathcal{M}_{\text {rel }}$ of relaxed microstructure parameters,

$$
\mathcal{M}_{\text {rel }}=\left\{\theta_{1}, \ldots, \theta_{6}, \varphi_{1}, \ldots, \varphi_{6}, \lambda, \lambda^{\prime}\right\},
$$

for which the coplanarity condition must be additionally enforced. This condition is adopted in the following form, cf. Eq. (11),

$$
(\mathbf{m} \times \mathbf{n}) \times\left(\mathbf{m}^{\prime} \times \mathbf{n}^{\prime}\right)=\mathbf{0},
$$

which is valid under the assumption that $\mathbf{m}$ and $\mathbf{n}$, and similarly $\mathbf{m}^{\prime}$ and $\mathbf{n}^{\prime}$, are not parallel. This condition, though not fully general, proved efficient in our computations.

Now, for a fixed microstructure $\mathcal{M}$, the strains and stresses in all phases are fully defined by the following strain-like parameters collected in the set $\mathcal{E}$,

$$
\mathcal{E}=\left\{\mathbf{F}_{1}, \mathbf{a}, \mathbf{a}^{\prime}, \mathbf{b}, \mathbf{b}^{\prime}, \mathbf{c}, \mathbf{c}^{\prime}\right\},
$$

provided that the compatibility constraint (17) is satisfied. Here, in order to constrain the rigid-body rotation, the deformation gradient $\mathbf{F}_{1}=\mathbf{F}_{1}^{e}$ is taken as a symmetric tensor, $\mathbf{F}_{1}=\mathbf{F}_{1}^{T}$, with six independent components included in $\mathcal{E}$. The deformation gradients $\mathbf{F}_{2}, \mathbf{F}_{2}^{\prime}, \mathbf{F}_{3}, \mathbf{F}_{21}, \mathbf{F}_{22}, \mathbf{F}_{21}^{\prime}$ and $\mathbf{F}_{22}^{\prime}$ are easily determined in terms of strain-like parameters $\mathcal{E}$ using the compatibility conditions (16) and (19), and the averaging rules (18).

We can now introduce the total elastic strain energy $W_{\text {total }}$ for the X-microstructure as a weighted sum of the local energy densities $W_{i}$ in the four domains,

$$
W_{\mathrm{total}}=\sum_{i=1}^{4} \omega_{i} W_{i},
$$

where $\omega_{i}$ are the weights, to be specified later, the energy in the twinned martensite domains is given by

$$
W_{2}=\lambda W_{21}+(1-\lambda) W_{22}, \quad W_{4}=W_{2}^{\prime}=\lambda^{\prime} W_{21}^{\prime}+\left(1-\lambda^{\prime}\right) W_{22}^{\prime},
$$

and the notation $W_{4}=W_{2}^{\prime}$ has been introduced for convenience.

The total elastic strain energy $W_{\text {total }}$ can now be minimized simultaneously with respect to the strain-like and microstructure parameters, namely

$$
\min _{\mathcal{E}, \mathcal{M}_{\text {rel }}} W_{\text {total }} \text { subject to constraints (17) and (22). }
$$

It can be shown that minimization of $W_{\text {total }}$ with respect to the vectors $\mathbf{a}, \ldots, \mathbf{c}^{\prime}$ in $\mathcal{E}$ enforces equilibrium conditions (15) at the corresponding interfaces. Furthermore, minimization of $W_{\text {total }}$ with respect to $\mathbf{F}_{1}$ enforces the average first Piola-Kirchhoff stress $\mathbf{S}_{\mathrm{av}}$ to be equal to zero, where $\mathbf{S}_{\mathrm{av}}=\sum_{i=1}^{4} \omega_{i} \mathbf{S}_{i}$ and $\mathbf{S}_{i}=\partial W_{i} / \partial \mathbf{F}_{i}$.

At the same time, by minimizing $W_{\text {total }}$ with respect to the microstructural parameters $\mathcal{M}_{\text {rel }}$, we are looking for low-energy microstructures. Note that the total elastic strain energy $W_{\text {total }}$ is expected to be a non-convex function of microstructure parameters, and thus, multiple local minima are expected. Accordingly, our aim here is to explore the local minima in the neighborhood of the (incompatible) candidate X-microstructures studied in Sect. 2.4. Specifically, the minimization problem (26) is solved for each candidate X-microstructure, and the interface orientations (obtained from the crystallographic theory) in the corresponding V-microstructures are used as the starting point in the minimization algorithm.

The specific minimization algorithm used in this work involves two stages. In the first stage, a sequence of unconstrained minimization problems is solved (using the BFGS method), in which the constraints (17) and (22) are enforced approximately using the penalty method with gradually increasing penalty parameter. In the second stage, the parameters obtained in the first stage are used as an initial guess for the constrained 
minimization problem that is solved using the interior point method implemented in the FindMinimum function of the Mathematica package (http://www.wolfram.com).

Initial numerical investigations have revealed that the effect of variable orientation of the twinning planes is negligible, i.e., the minimum has always been found for $\mathbf{I}$ and $\mathbf{I}^{\prime}$ equal (up to the numerical error) to the initial values corresponding to the crystallographic theory. Accordingly, in the actual computations, the energy is minimized with respect to a reduced set of microstructure parameters $\mathcal{M}^{*}=\left\{\mathbf{m}, \mathbf{m}^{\prime}, \mathbf{n}, \mathbf{n}^{\prime}, \lambda, \lambda^{\prime}\right\}$, while $\mathbf{l}$ and $\mathbf{I}^{\prime}$ are taken from the crystallographic theory.

\section{Results: X-microstructures in CuAINi}

Minimization of the total elastic strain energy $W_{\text {total }}$ has been performed for all 528 crystallographically distinct candidate X-microstructures, see Sect. 2.4. At this stage, constant weights $\omega_{i}=\frac{1}{4}$, independent of the microstructure $\mathcal{M}$, have been adopted, cf. Eq. (24). A more realistic assumption is discussed later.

We start the analysis of the results by pointing out some general observations. Next, a more detailed discussion is presented for the microstructures characterized by relatively low energy.

First of all, we note that the number of distinct $\mathrm{X}$-microstructures obtained by minimization of the elastic strain energy is smaller than the number of considered candidate $\mathrm{X}$-microstructures. The reason is that in many cases the same solution has been obtained for more than one candidate $\mathrm{X}$-microstructure used as a starting point in the minimization algorithm. This may be related to the complex energy landscape in the multidimensional space of microstructure parameters, but also to the properties of the adopted minimization procedure which does not guarantee that all local minima of the elastic strain energy are found.

Considering the characteristic shapes and arrangement of the domains, two types of X-microstructures have been observed. Microstructures of the first type, see Fig. $6 a$ and $b$, have been obtained for the variant triples $(1,3,5)$ and $(1,3,6)$. Note that, in view of the assumption $\omega_{i}=\frac{1}{4}$, the two microstructures shown in Fig. 6a and $b$ have identical energy and are thus equivalent. Similarly, the microstructures corresponding to the variant triples $(1,3,3)$ and $(1,3,4)$ are shown in Fig. $6 \mathrm{c}$ and d. The characteristic feature of the microstructures of the second type is that the two M-MM interfaces are nearly parallel, so that either domain $\Omega_{1}$ or domain $\Omega_{3}$ occupies, approximately, a half-plane. Note that the microstructures observed in CuAlNi by Seiner et al. [22] are exclusively of the type shown in Fig. 6a.

In Table 1, we provide the microstructure parameters and the elastic strain energy $W_{\text {total }}$ obtained for four selected microstructures. The twin fractions $\lambda_{J}=1-\lambda$ and $\lambda_{K}^{\prime}=1-\lambda^{\prime}$ correspond to the volume fractions of variants $J$ and $K$, respectively. As a reference, the microstructure parameters of the closest candidate $\mathrm{X}$-microstructure (as following from the crystallographic theory) are provided in Table 1 for each case along with the corresponding incompatibility indicators $\phi$ and $\psi$. The minimum, maximum, and average values of equivalent (Huber-von Mises) stresses $\sigma_{\text {eq }}$ have also been included in Table 1.

The microstructure characterized by the lowest energy has been obtained for the variant triple $(I, J, K)=$ $(1,3,4)$, i.e., it is of the type corresponding to Fig. $6 c$, d. In this microstructure, the twinning plane normal $\mathbf{l}$ is approximately perpendicular to M-MM interface normal $\mathbf{n}$, so it can be classified as a " $\lambda$-interface", see Remark 1. Further, in Table 1 we report two X-microstructures corresponding to Fig. 6a, b which are characterized by the lowest energy among the " $\lambda$-interfaces" and "X-interfaces". Both microstructures involve the $(1,3,6)$ variant triple.

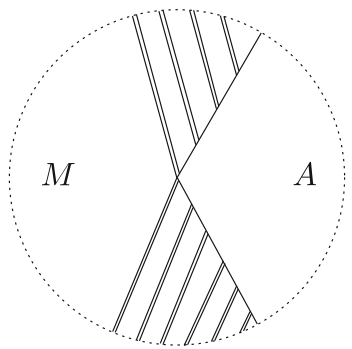

(a)

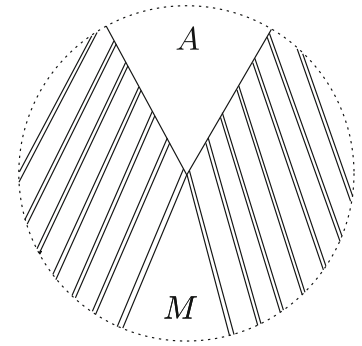

(b)

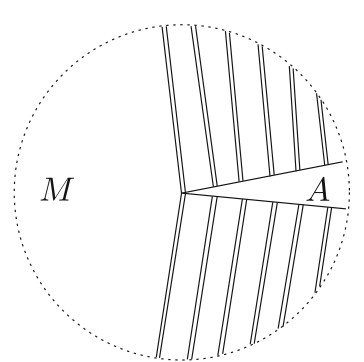

(c)

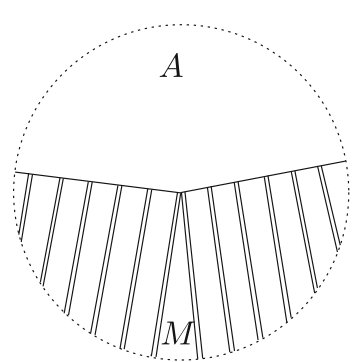

(d)

Fig. 6 Types of X-microstructures obtained by energy minimization (schematic): a, b variant triples $(1,3,5)$ and $(1,3,6)$; c, d variant triples $(1,3,3)$ and $(1,3,4)$ 
Table 1 Parameters of selected low-energy X-microstructures

\begin{tabular}{|c|c|c|c|c|}
\hline & Crystallographic theory & Energy minimization & & \\
\hline & & $\omega_{i}=1 / 4$ & $\omega_{i}=\alpha_{i} /(2 \pi)(1)$ & $\omega_{i}=\alpha_{i} /(2 \pi)(2)$ \\
\hline Microstruc & are $1 .(I, J, K)=(1,3,4),{ }^{\prime} \lambda$-in & rface': & & \\
\hline$\lambda_{J}$ & 0.3008 & 0.2923 & 0.2659 & 0.2404 \\
\hline 1 & $(0.2282,0.6885,0.6885)$ & - & - & - \\
\hline $\mathbf{n}$ & $(0.0207,-0.6417,0.7666)$ & $(0.0266,-0.6373,0.7702)$ & $(-0.0241,0.6370,-0.7705)$ & $(0.0069,-0.6433,0.7656)$ \\
\hline $\mathbf{m}$ & $(-0.7304,0.6679,-0.1430)$ & $(-0.7264,0.6729,-0.1399)$ & $(0.7223,-0.6721,0.1628)$ & $(-0.7193,0.6722,-0.1756)$ \\
\hline$\lambda_{K}^{\prime}$ & 0.3008 & 0.2861 & 0.2613 & 0.2385 \\
\hline $\mathrm{I}^{\mathrm{N}}$ & $(0.2282,-0.6885,0.6885)$ & - & - & - \\
\hline $\mathbf{n}^{\prime}$ & $(0.2282,-0.6885,0.6885)$ & $(0.2003,-0.7020,0.6834)$ & $(0.2039,-0.7014,0.6830)$ & $(0.1896,-0.7068,0.6816)$ \\
\hline $\mathbf{m}^{\prime}$ & $(0.6345,-0.7276,0.2607)$ & $(0.6398,-0.7166,0.2779)$ & $(0.6430,-0.7108,0.2850)$ & $(-0.6468,0.7084,-0.2827)$ \\
\hline$\phi$ & $5.321^{\circ}$ & & & \\
\hline$\psi$ & $0.126^{\circ}$ & & & \\
\hline$W_{\text {total }}$ & & $0.0482\left[\mathrm{MJ} / \mathrm{m}^{3}\right]$ & $0.0190\left[\mathrm{MJ} / \mathrm{m}^{3}\right]$ & $0.0323\left[\mathrm{MJ} / \mathrm{m}^{3}\right]$ \\
\hline$\sigma_{\mathrm{eq}}$ & & $43 / 201 / 116[\mathrm{MPa}]$ & 8/199/106 [MPa] & 55/336/135 [MPa] \\
\hline Microstruc & are 2. $(I, J, K)=(1,3,6)$, ' $\lambda$-in & rface': & & \\
\hline$\lambda_{J}$ & 0.2902 & 0.2838 & 0.2856 & 0.2796 \\
\hline 1 & $(0,-0.7071,0.7071)$ & - & - & - \\
\hline $\mathbf{n}$ & $(-0.2272,-0.6216,-0.7497)$ & $(-0.2295,-0.6270,-0.7444)$ & $(0.2289,0.6272,0.7445)$ & $(-0.2310,-0.6260,-0.7448)$ \\
\hline $\mathbf{m}$ & $(0.6350,0.7486,0.1908)$ & $(0.6363,0.7466,0.1943)$ & $(0.6355,0.7468,0.1962)$ & $(0.6390,0.7451,0.1911)$ \\
\hline$\lambda_{K}^{\prime}$ & 0.2902 & 0.3040 & 0.3033 & 0.3067 \\
\hline $1^{\wedge}$ & $(0.7071,0.7071,0)$ & - & - & - \\
\hline $\mathbf{n}^{\prime}$ & $(-0.7071,-0.7071,0)$ & $(-0.7052,-0.7090,0.0079)$ & $(-0.7048,-0.7094,0.0061)$ & $(-0.7063,-0.7078,0.0081)$ \\
\hline $\mathbf{m}^{\prime}$ & $(0.2580,0.6497,0.7151)$ & $(0.2572,0.6429,0.7215)$ & $(-0.2558,-0.6426,-0.7223)$ & $(0.2598,0.6424,0.7210)$ \\
\hline$\phi$ & $1.368^{\circ}$ & & & \\
\hline$\psi$ & $0.173^{\circ}$ & & & \\
\hline$W_{\text {total }}$ & & $0.0502\left[\mathrm{MJ} / \mathrm{m}^{3}\right]$ & $0.0377\left[\mathrm{MJ} / \mathrm{m}^{3}\right]$ & $0.0402\left[\mathrm{MJ} / \mathrm{m}^{3}\right]$ \\
\hline$\sigma_{\mathrm{eq}}$ & & 91/148/115 [MPa] & 47/198/133 [MPa] & 46/181/102 [MPa] \\
\hline Microstruc & are 3. $(I, J, K)=(1,3,6)$, 'X-in & rface': & & \\
\hline$\lambda_{J}$ & 0.2902 & 0.2786 & 0.2747 & 0.2808 \\
\hline 1 & $(0,-0.7071,0.7071)$ & - & - & - \\
\hline $\mathbf{n}$ & $(0,-0.7071,0.7071)$ & $(0.0211,-0.6935,0.7202)$ & $(0.0251,-0.6935,0.7200)$ & $(-0.0142,0.6965,-0.7174)$ \\
\hline $\mathbf{m}$ & $(-0.7151,0.6497,-0.2580)$ & $(-0.7092,0.6611,-0.2449)$ & $(-0.7090,0.6628,-0.2409)$ & $(-0.7096,0.6592,-0.2488)$ \\
\hline$\lambda_{K}^{\prime}$ & 0.3008 & 0.3138 & 0.3181 & 0.3127 \\
\hline $1^{\Lambda}$ & $(0.6885,-0.6885,0.2282)$ & - & - & - \\
\hline $\mathbf{n}^{\prime}$ & $(0.6885,-0.6885,0.2282)$ & $(0.6949,-0.6694,0.2629)$ & $(0.6950,-0.6709,0.2587)$ & $(0.6947,-0.6679,0.2671)$ \\
\hline $\mathbf{m}^{\prime}$ & $(-0.2607,0.7276,-0.6345)$ & $(-0.2468,0.7404,-0.6252)$ & $(-0.2529,0.7411,-0.6219)$ & $(0.2398,-0.7423,0.6257)$ \\
\hline$\phi$ & $8.542^{\circ}$ & & & \\
\hline$\psi$ & $0.216^{\circ}$ & & & \\
\hline$W_{\text {total }}$ & & $0.0749\left[\mathrm{MJ} / \mathrm{m}^{3}\right]$ & $0.0569\left[\mathrm{MJ} / \mathrm{m}^{3}\right]$ & $0.0484\left[\mathrm{MJ} / \mathrm{m}^{3}\right]$ \\
\hline$\sigma_{\mathrm{eq}}$ & & $128 / 179 / 153[\mathrm{MPa}]$ & 68/252/133 [MPa] & 49/302/173 [MPa] \\
\hline Microstruc & are 4. $(I, J, K)=(3,1,5),{ }^{\prime} \mathrm{X}$-in & face': & & \\
\hline$\lambda_{J}$ & 0.3008 & 0.2979 & 0.3001 & 0.2890 \\
\hline 1 & $(0.2282,0.6885,0.6885)$ & - & - & - \\
\hline $\mathbf{n}$ & $(0.2282,0.6885,0.6885)$ & $(0.2106,0.6790,0.7033)$ & $(-0.2139,-0.6785,-0.7028)$ & $(0.2087,0.6811,0.7018)$ \\
\hline $\mathbf{m}$ & $(-0.6345,-0.2607,-0.7276)$ & $(-0.6386,-0.2720,-0.7199)$ & $(-0.6400,-0.2730,-0.7183)$ & $(-0.6394,-0.2765,-0.7174)$ \\
\hline$\lambda_{K}^{\prime}$ & 0.3008 & 0.2979 & 0.3009 & 0.2948 \\
\hline $\mathrm{I}^{\mathrm{\Lambda}}$ & $(0.6885,0.2282,0.6885)$ & - & - & - \\
\hline $\mathbf{n}^{\prime}$ & $(0.6885,0.2282,0.6885)$ & $(0.6790,0.2106,0.7033)$ & $(0.6789,0.2140,0.7024)$ & $(0.6812,0.2136,0.7002)$ \\
\hline $\mathbf{m}^{\prime}$ & $(-0.2607,-0.6345,-0.7276)$ & $(-0.2720,-0.6386,-0.7199)$ & $(0.2728,0.6397,0.7186)$ & $(-0.2759,-0.6373,-0.7196)$ \\
\hline$\phi$ & $7.275^{\circ}$ & & & \\
\hline$\psi$ & $0.247^{\circ}$ & & & \\
\hline$W_{\text {total }}$ & & $0.1840\left[\mathrm{MJ} / \mathrm{m}^{3}\right]$ & $0.1080\left[\mathrm{MJ} / \mathrm{m}^{3}\right]$ & $0.1247\left[\mathrm{MJ} / \mathrm{m}^{3}\right]$ \\
\hline$\sigma_{\mathrm{eq}}$ & & 208/259/233 [MPa] & 75/369/269 [MPa] & 87/445/202 [MPa] \\
\hline
\end{tabular}

Finally, microstructure 4 in Table 1 corresponds to the microstructure that has been actually observed in experiments [21,22]. This microstructure has been identified in [21] to be composed of martensite variants ${ }^{2}$ (3, $1,5)$. The variant triple $(3,1,5)$ is crystallographically equivalent to the triple $(1,3,5)$, so the $\mathrm{X}$-microstructure corresponds to that shown in Fig. 6a, as observed experimentally [21,22]. Further, in agreement with experimental observations, microstructure 4 involves type II twins in domains $\Omega_{2}$ and $\Omega_{2}^{\prime}$ and the M-MM interfaces

\footnotetext{
${ }^{2}$ In the numbering of variants adopted in [21], these variants are actually numbered as $(6,4,2)$.
} 
are nearly parallel to the corresponding twinning planes, i.e., it is an "X-interface". The incompatibility indicator $\phi=7.275^{\circ}$ agrees well with the value reported in [21], and so does an alternative incompatibility indicator, namely the angle between vectors $\mathbf{m} \times \mathbf{m}^{\prime}$ and $\mathbf{n} \times \mathbf{n}^{\prime}$, which is equal to $2.252^{\circ}$. The incompatibility indicator $\psi=0.247^{\circ}$ in Table 1 differs from the value of $\psi=6.6^{\circ}$ provided by Seiner et al. [21]; however, the latter value is a misprint ${ }^{3}$ and the correct one is that given in Table 1.

For the four microstructures listed in Table 1, we have additionally analyzed the effect of the weights $\omega_{i}$ on the resulting microstructures. Specifically, the weight $\omega_{i}$ has been assumed proportional to the wedge angle $\alpha_{i}$ between the two interfaces delimiting the domain $\Omega_{i}$, namely $\omega_{i}=\alpha_{i} /(2 \pi)$. The weights $\omega_{i}$ defined in this way can be interpreted as the volume fractions of the corresponding domains. Note that each solution obtained using a constant weight $\omega_{i}=\frac{1}{4}$ generates now two distinct solutions with different arrangement of the domains, cf. Fig. 6 . The results are also included in Table 1.

The most important observation following from the analysis of the results in Table 1 is that microstructure parameters obtained by energy minimization are very close to the respective parameters predicted by the crystallographic theory for individual interfaces. The interface normal vectors differ by at most $2.5^{\circ}$, and in most cases, the difference is below $1^{\circ}$. As the twin fractions $\lambda_{J}$ and $\lambda_{K}^{\prime}$ are concerned, the difference is somewhat more pronounced reaching 0.06 (in absolute values) for the case of $(I, J, K)=(1,3,4)$ in Table 1 , but for the remaining three microstructures the difference is below 0.02 .

The elastic strain energy $W_{\text {total }}$ is an overall measure of elastic strains and stresses that are necessary to achieve compatibility at interfaces. However, it is also of interest to examine the local stresses in individual phases (we remind here that the local stresses in the individual phases are self-equilibrated, i.e., they average to zero). The local Cauchy stresses have been calculated for the six phases involved (austenite in domain $\Omega_{1}$, martensite in domain $\Omega_{3}$, and individual martensite variants in the twinned domains $\Omega_{2}$ and $\Omega_{2}^{\prime}$ ), along with the corresponding equivalent Huber-von Mises stresses $\sigma_{\mathrm{y}}$ (though, clearly, the relevance of this stress invariant is restricted at the crystal lattice scale). Next, the minimum, maximum, and average values of the equivalent stress $\sigma_{\mathrm{y}}$ have been determined for each microstructure and included in Table 1 (here, the average is a simple arithmetic average). The obtained stress levels seem realistic in a sense that the maximum values, varying between 148 and $445 \mathrm{MPa}$, do not exceed the macroscopic stresses typically observed, e.g., in uniaxial loading experiments on CuAlNi single crystals.

It is also seen from Table 1 that the effect of the variable weights $\omega_{i}=\alpha_{i} /(2 \pi)$ on the obtained microstructure parameters is small. Accordingly, we conclude that the use of constant weights $\omega_{i}=\frac{1}{4}$ is sufficient for our purposes. At the same time, the minimization problem is solved more easily, and the number of microstructures is reduced as the arrangement of the domains need not be considered. In the remaining part, we only discuss the results corresponding to $\omega_{i}=\frac{1}{4}$.

The minimum energies obtained for all 528 candidate X-microstructures vary between 0.048 and 14.1 $\mathrm{MJ} / \mathrm{m}^{3}$. We have tried to find a relationship between these energies and the incompatibility indicators $\phi$ and $\psi$. However, the data corresponding to all candidate X-microstructures exhibits large scatter. Analysis of the complete set of data is also difficult because quite often, but without a visible pattern, the same final microstructure (and identical energy) has been obtained for several candidate X-microstructures.

A more detailed analysis has thus been carried out for 31 microstructures that are characterized by the lowest incompatibility indicators, $\phi<20^{\circ}$ and $\psi<2^{\circ}$, for which the energy is $W_{\text {total }}<2 \mathrm{MJ} / \mathrm{m}^{3}$. Clearly, only the microstructures of the lowest energy are likely to appear in practice.

As already mentioned, the same solution (microstructure minimizing the energy) may correspond to several starting point candidate $\mathrm{X}$-microstructures. In the case of the selected 31 microstructures, this could be studied in more detail. We have observed that there are four candidate X-microstructures that usually give the same solution, and these have identical microstructural parameters except the orientations of the M-MM interfaces (we have $\mathbf{n}=\mathbf{l}$ or $\mathbf{n} \cdot \mathbf{l} \approx 0$; and similarly for $\mathbf{n}^{\prime}$ and $\mathbf{l}^{\prime}$ ). The $\mathrm{X}$-microstructure found by our energy minimization approach is then in the neighborhood of the candidate $\mathrm{X}$-microstructure that is characterized by the lowest incompatibility indicator $\phi$ out of the four candidate X-microstructures.

Figure 7 shows the elastic strain energy $W_{\text {total }}$ of the 31 selected $X$-microstructures as a function of the incompatibility indicators $\phi$ and $\psi$ determined for the closest candidate $\mathrm{X}$-microstructure. The red dots denote variant triples $(1,3,3)$ and $(1,3,4)$ with $\lambda_{J} \approx 0.3$ and $\lambda_{K}^{\prime} \approx 0.3$. Similarly, the green squares denote variant triples $(1,3,5)$ and $(1,3,6)$ with $\lambda_{J} \approx 0.3$ and $\lambda_{K}^{\prime} \approx 0.3$. The blue diamonds indicate the microstructures with $\lambda_{J} \approx 0.7$ or $\lambda_{K}^{\prime} \approx 0.7$ formed by either of the variant triples. It is seen that low-energy X-microstructures are mostly characterized by $\lambda_{J} \approx 0.3$ and $\lambda_{K}^{\prime} \approx 0.3$.

\footnotetext{
${ }^{3}$ H. Seiner, private communication (2011).
} 
(a)

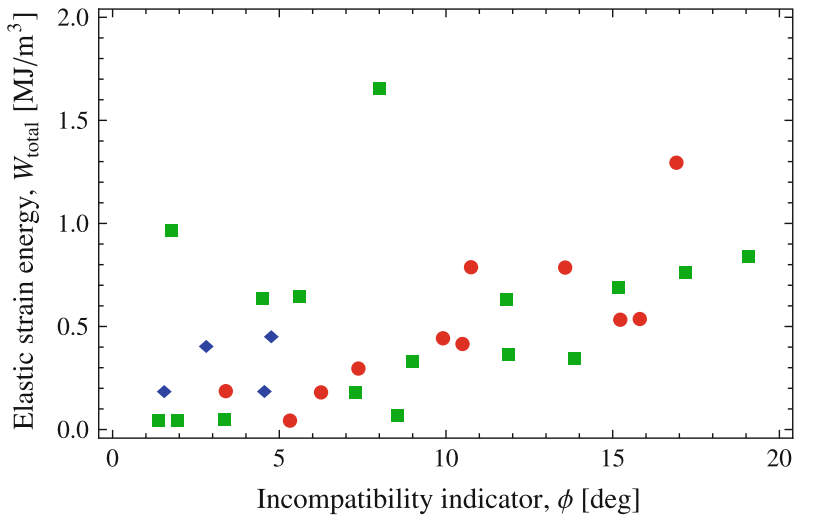

(b)

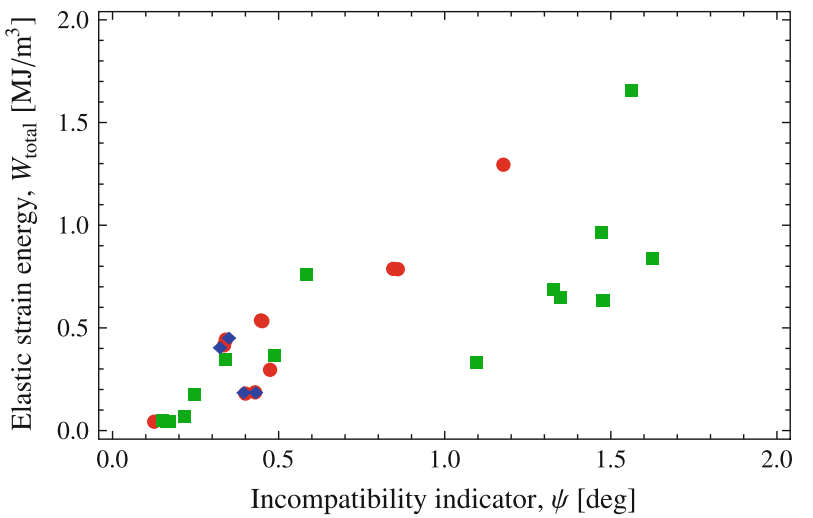

Fig. 7 Elastic strain energy $W_{\text {total }}$ as function of $\mathbf{a}$ incompatibility indicator $\phi$ and $\mathbf{b}$ incompatibility indicator $\psi$

Comparing Fig. 7a and $\mathrm{b}$ it is seen that the elastic strain energy $W_{\text {total }}$ shows higher correlation with the incompatibility indicator $\psi$ than with $\phi$. Further, from Fig. 5, it follows that there exist candidate X-microstructures characterized by very low values of $\phi$ and at the same time by relatively high values of $\psi$, and the corresponding X-microstructures, not included in Fig. 7, are also characterized by high values of $W_{\text {total }}$. This leads to a conclusion that the incompatibility indicator $\psi$ alone can be used to select those candidate $\mathrm{X}$-microstructures which yield low-energy (almost compatible) $\mathrm{X}$-microstructures. Clearly, the analysis above and the conclusion apply to the CuAlNi alloy undergoing the cubic-to-orthorhombic transformation, and other shape memory alloys would require a separate study.

\section{Conclusion}

An energy-based approach has been developed for the analysis of almost compatible martensitic microstructures. As an application, the X-microstructures have been studied in the CuAlNi shape memory alloy undergoing the cubic-to-orthorhombic transformation. The approach is general and could also be applied to other microstructures, e.g., to wedge microstructures.

The set of 528 crystallographically distinct candidate X-microstructures in the cubic-to-orthorhombic transformation has been determined by considering the X-microstructure as an assembly of two V-microstructures. Two incompatibility indicators have been introduced, and it has been verified numerically that these indicators are never simultaneously equal to zero, so that none of the candidate X-microstructures is compatible. The conclusion of this part of the paper is that the elastic strains are necessarily present in the experimentally observed X-microstructures.

Accordingly, almost compatible X-microstructures have been studied by assuming that the deformation gradient comprises the elastic and transformation parts and is piecewise-constant. The total elastic strain energy has been expressed in terms of the microstructure parameters; specifically, the interface normals and 
twin fractions. Local minima of the energy have next been searched in the neighborhood of all candidate Xmicrostructures in CuAlNi alloy. As a result, several low-energy X-microstructures have been found, for which the obtained microstructure parameters only slightly deviate from the corresponding parameters predicted by the crystallographic theory. It has also been shown that the incompatibility indicator $\psi$ alone can be used to select the candidate $\mathrm{X}$-microstructures which yield low-energy $\mathrm{X}$-microstructures. Further, significant correlation exists between the incompatibility indicator $\psi$ of the candidate $\mathrm{X}$-microstructure and the total elastic strain energy of the corresponding almost compatible, low-energy X-microstructure.

The assumption that the deformation gradient is uniform within each of the domains forming the $\mathrm{X}$-microstructure implies that the boundary conditions adequate for a specimen are not accounted for. Accordingly, the analysis is most relevant in the vicinity of the intersection line. However, this simplifying assumption allows us to carry out a systematic study for all 528 candidate X-microstructures.

The analysis of Seiner et al. [21] and Glatz et al. [9] indicates that the X-microstructure formed within a bar (as observed experimentally) does not correspond to a local minimum of elastic strain energy. Accordingly, formation and propagation of the X-microstructure cannot be explained by the tendency of the material to minimize its free energy only. Thus, it seems that, in order to understand the actual mechanism of formation and propagation of the $\mathrm{X}$-microstructure, which is unknown to date, it is crucial to consider energy dissipation and an incremental energy minimization scheme, possibly including the interfacial energy effects, cf. [16] and references therein. The characteristic elastic strain energy, which can be determined using the approach proposed in this work, is one of the factors influencing selection of the actual X-microstructure that forms in specific conditions.

Acknowledgments This work has been partially supported by the Ministry of Science and Higher Education in Poland under Grant no. N N501 071935.

Open Access This article is distributed under the terms of the Creative Commons Attribution Noncommercial License which permits any noncommercial use, distribution, and reproduction in any medium, provided the original author(s) and source are credited.

\section{References}

1. Balandraud, X., Delpueyo, D., Grediac, M., Zanzotto, G.: Almost compatible microstructures in shape memory alloys. Acta Mater. 58, 4559-4577 (2010)

2. Balandraud, X., Zanzotto, G.: Stressed microstructures in thermally induced M9R-M18R martensites. J. Mech. Phys. Solids 55, 194-224 (2007)

3. Ball, J.M., James, R.D.: Fine phase mixtures as minimizers of energy. Arch. Ration. Mech. Anal. 100, 13-50 (1987)

4. Basinski, Z.S., Christian, J.W.: Experiments on the martensitic transformation in single crystals of indium-thalium alloys. Acta Metall. 2, 148-166 (1954)

5. Bhattacharya, K.: Microstructure of Martensite: Why it Forms and How it Gives Rise to the Shape-Memory Effect. Oxford University Press, Oxford (2003)

6. Bowles, J.S., MacKenzie, J.K.: The crystallography of martensitic transformations I and II. Acta Metall. 2, 129-137, 138-147 (1954)

7. Chu, C.: Hysteresis and microstructures: a study of biaxial loading on compound twins of copper-aluminum-nickel single crystals. Ph.D. thesis, University of Minnesota (1993)

8. Delville, R., Kasinathan, S., Zhang, Z., Van Humbeck, J., James, R.D., Schryvers, D.: Transmission electron microscopy study of phase compatibility in low hysteresis shape memory alloys. Philos. Mag. 90, 177-195 (2010)

9. Glatz, O., Seiner, H., Landa, M.: FEM modelling of elastically strained interfacial microstructures in Cu-Al-Ni single crystals. In: Sittner, P., Heller, L., Paidar, V. (eds.) ESOMAT 2009-The 8th European Symposium on Martensitic Transformations, p. 03006. EDP Sciences (2009). doi:10.1051/esomat/200903006

10. Hane, K.F.: Bulk and thin film microstructures in untwinned martensites. J. Mech. Phys. Solids 47, 1917-1939 (1999)

11. Hane, K.F., Shield, T.W.: Microstructure in a cubic to orthorhombic transition. J. Elast. 59, 267-318 (2000)

12. Khachaturyan, A.G.: Theory of Structural Transformations in Solids. Wiley, New York (1983)

13. Kohn, R.V., Müller, S.: Branching of twins near an austenite-twinned-martensite interface. Philos. Mag. A 66(5), 697715 (1992)

14. Maciejewski, G., Stupkiewicz, S., Petryk, H.: Elastic micro-strain energy at the austenite-twinned martensite interface. Arch. Mech. 57, 277-297 (2005)

15. Novák, V., Šittner, P., Ignacová, S., Černoch, T.: Transformation behaviour of prism shaped shape memory alloy single crystals. Mater. Sci. Eng. A 438-440, 755-762 (2006)

16. Petryk, H., Stupkiewicz, S.: Interfacial energy and dissipation in martensitic phase transformations. Part I: Theory. J. Mech. Phys. Solids 58, 390-408 (2010)

17. Roytburd, A.L.: Martensitic transformation as a typical phase transformation in solids. In: Seitz, D., Turnbull, D. (eds.) Solid State Physics, vol. 33, pp. 317-380. Academic Press, New York (1978)

18. Roytburd, A.L.: Thermodynamics of polydomain heterostructures II. Effect of microstresses. J. Appl. Phys. 83(1), 239245 (1998) 
19. Ruddock, G.J.: A microstructure of martensite which is not a minimizer of energy: the X-interface. Arch. Ration. Mech. Anal. 127, 1-39 (1994)

20. Sedlak, P., Seiner, H., Landa, M., Novak, V., Sittner, P., Manosa, L.: Elastic constants of bec austenite and 2H orthorhombic martensite in CuAlNi shape memory alloy. Acta Mater. 53, 3643-3661 (2005)

21. Seiner, H., Glatz, O., Landa, M.: Interfacial microstructures in martensitic transitions: from optical observations to mathematical modelling. Int. J. Multiscale Comp. Eng. 7, 445-456 (2009)

22. Seiner, H., Sedlak, P., Landa, M.: Shape recovery mechanism observed in single crystals of Cu-Al-Ni shape memory alloy. Phase Trans. 81, 537-551 (2008)

23. Stupkiewicz, S.: The effect of stacking fault energy on the formation of stress-induced internally faulted martensite plates. Eur. J. Mech. A/Solids 23, 107-126 (2004)

24. Stupkiewicz, S., Maciejewski, G., Petryk, H.: Low-energy morphology of the interface layer between austenite and twinned martensite. Acta Mater. 55, 6292-6306 (2007)

25. Suezawa, M., Sumino, K.: Behaviour of elastic constants in Cu-Al-Ni alloy in the close vicinity of $M_{s}$-point. Scr. Metall. 10, 789-792 (1976)

26. Wechsler, M.S., Lieberman, D.S., Read, T.A.: On the theory of the formation of martensite. Trans. AIME J. Metals 197, 15031515 (1953)

27. Yasunaga, M., Funatsu, Y., Kojima, S., Otsuka, K., Suzuki, T.: Measurement of elastic constants. Scr. Metall. 17, 1091$1094(1983)$

28. Zhang, Z., James, R.D., Muller, S.: Energy barriers and hysteresis in martensitic phase transformations. Acta Mater. 57, 4332-4352 (2009) 